\title{
Optimization of Insulator Shape Using GA and HGAPSO Methods
}

\author{
M. R. Salimian, and H. Javadi
}

\begin{abstract}
The flashover voltage of insulator is relative to the distribution of the electric field. The distribution of the electric field on the insulator is contributed to the shape of insulator. For this, we must optimize the shape of insulator. In this paper, we use FEM, GA and HGAPSO for optimizing the shape of insulator. The tangential electric field and the area of insulator are optimized by GA and HGAPSO. Then, the results obtained from GA and HGAPSO are compared with each other.
\end{abstract}

Index Terms-FEM, GA, HGAPSO, insulator, electric field.

\section{INTRODUCTION}

$\mathrm{T}$ HE flashover voltage is an important parameter of insulator. The low flashover voltage may be caused to electric discharge. The flashover voltage of insulator can be increased by optimizing the shape of insulator. Numerical methods for optimizing the shape of insulator are presented in [1-3]. These methods are complex and may find local optimum.

CSM [4-7] and DAGA [8-9] are used in [10] for optimizing the shape of Taiwan power insulator. CSM which is used for computing the tangential electric field is not accurate. DAGA which is used for optimizing is relative to initial population. For this, the final shape of insulator is similar to the initial shape.

In this paper, the average and variance of tangential electric field and the area of insulator are optimized by GA and HGAPSO. Finite element method (FEM) [11] is used for computing the tangential electric field of insulator. At the end, the optimal design obtained from GA and HGAPSO are compared with each other and with original Taiwan power company insulator.

\section{OBJECTIVE FUNCTION}

In this paper, the shape of Taiwan power company insulator which is shown in Fig 1 has been optimized. 41 control points are considered for optimizing the shape of insulator. Search space of optimization is between $\pm 1 \mathrm{~cm}$ of the initial state of control points. Cubic spline function is used for smoothing the

M. R. SALIMAN is with the Department of Electrical Engineering, Faculty of Shahid Abbaspour, Shahid Beheshti University, Tehran, Iran (email: bmsalimian@gmail.com).

H. JAVADI is with the Department of Electrical Engineering, Faculty of Shahid Abbaspour, Shahid Beheshti University, Tehran, Iran (e-mail: javadih@yahoo.com). shape of insulator. Tangential electric field and the area of insulator are optimized. The objective function is presented in [1].

objectivefunction $=a_{1} E_{\text {ave }}+a_{2} \sigma_{E}+a_{3} A_{s}$

$E_{\text {ave }}=\frac{\sum_{i=1}^{41} E_{i}}{41}$

$\sigma_{E}=\sqrt{\frac{\sum_{i=1}^{41}\left(E_{i}-E_{\text {ave }}\right)^{2}}{41}}$

constraints,

$y_{j}^{\text {Down }}-y_{j}^{U p}<0$

$y_{j}^{\text {Down }} \geq y_{\text {Min }}$

$y_{i}^{U p}-y_{i+1}^{U p}>0$

$N_{\text {extreme }} \leq N_{\max }$

$A_{s}$ is the area of insulator shown in Fig 1.

$y_{j}^{U p}$ shows the altitude of the jth control points on the upper surface of insulator.

$y_{j}^{D o w n}$ shows the altitude of the jth control point on the lower surface of insulator.

$N_{\text {extreme }}$ shows the number of extremes on the lower surface of insulator.

$E_{i}$ is the tangential electric field of the control point $\mathrm{i}$. 


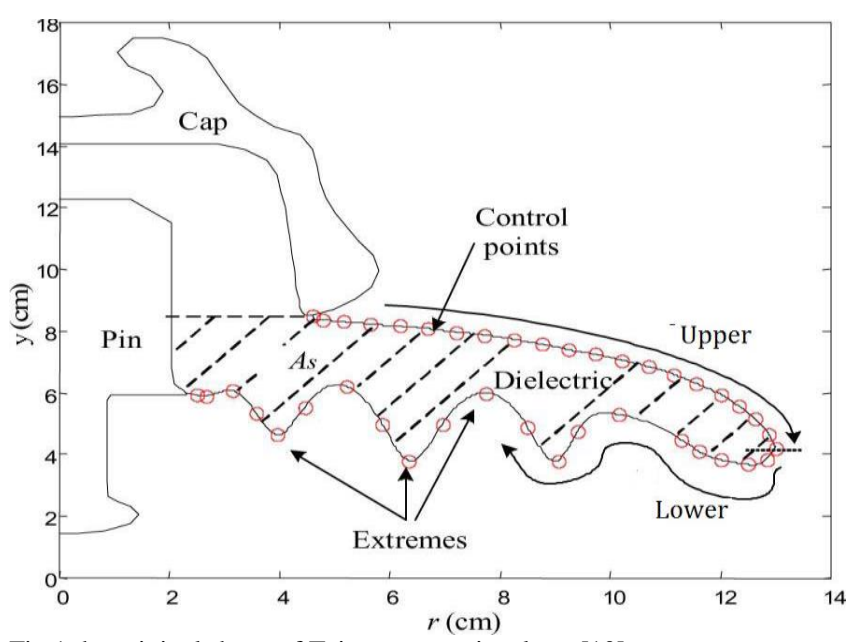

Fig.1 the original shape of Taiwan power insulator [10]

\section{HYBRID GA AND PSO (HGAPSO)}

This method is compound of GA and PSO. PSO is replaced by mutation in GA and change the position of population members by (8).

$X_{j}^{n}=X_{j}^{n-1}+v e l_{j}^{n}$

$\operatorname{vel}_{j}^{n}=w_{n} \times \operatorname{vel}_{j}^{n-1}+c \times R \otimes\left(\right.$ Gbest $\left.-X_{j}^{n-1}\right)$

$w_{n}$ is the inertia of the nth generation which is changed from 0.9 to 0.4 .

$R$ is a random vector.

Gbestis the best member of population.

$X_{j}^{n}$ is the position of the jth member in the nth generation.

cis a constant which is considered as 2 .

\section{Simulation Result}

MATLAB PDE toolbox [12] is used for computing the tangential electric field of insulator by FEM method. HGAPSO and GA which are coding by MATLAB are used for minimizing objective function. The population size, crossover fraction and the maximum number of generation are considered as $61,0.8$, and 30 , respectively. The permittivity of dielectric and the voltage of electrode are considered as 2.5 and 1 p.u, respectively.

The results obtained from HGAPSO and GA for $y_{\text {Min }}=$ $2.45 \mathrm{~cm}$ and $a_{3}=0.166$ are presented in Fig 2 and 3, respectively. The optimal design of of insulator obtained from GA and HGAPSO which are shown in Fig 4-5 are compared with original insulator in Fig 6-7, respectively. The minimum of objective function obtained from HGAPSO is equal to 14.3268 which is smaller than the results obtained from GA that is equal to 14.6213 .

The average and variance of tangential electric field and the area of insulator are compared for different cases in Table 1.

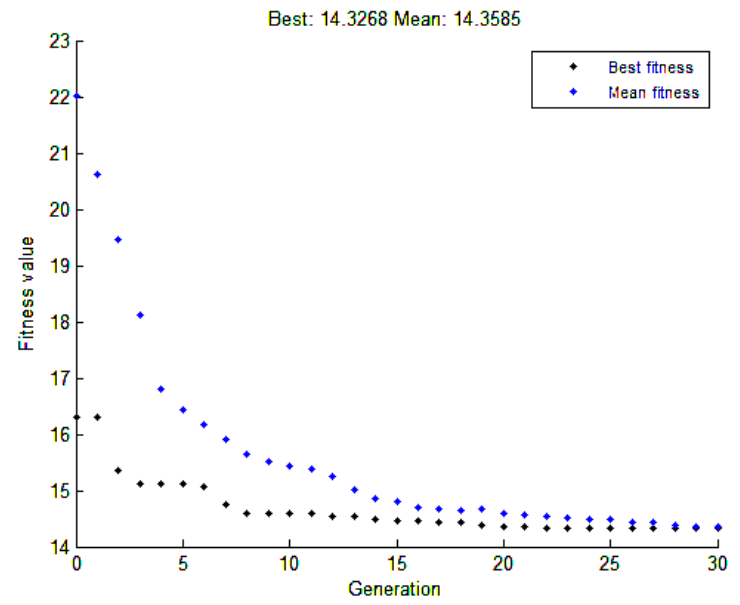

Fig.2 the minimum values of objective function obtained from HGAPSO $\left(a_{3}=0.166\right)$

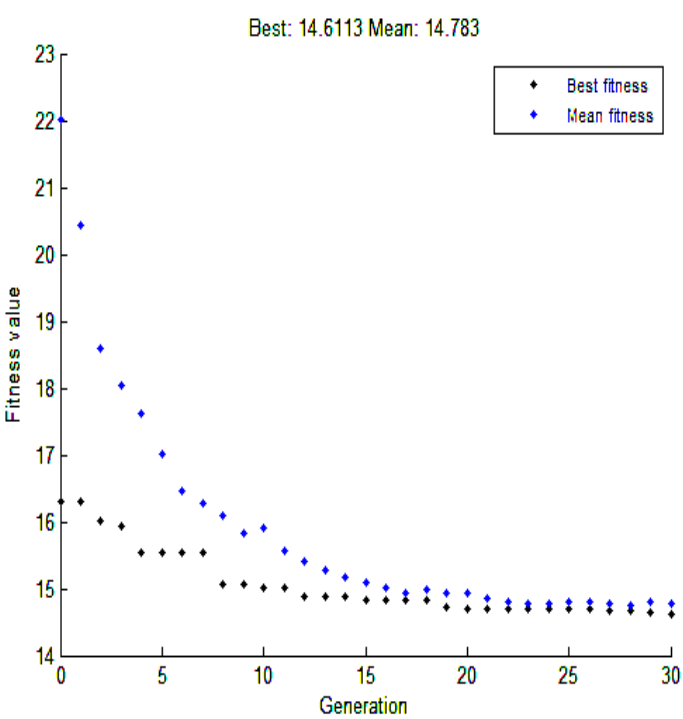

Fig. 3 the minimum values of objective function obtained from GA $\left(a_{3}=\right.$ $0.166)$

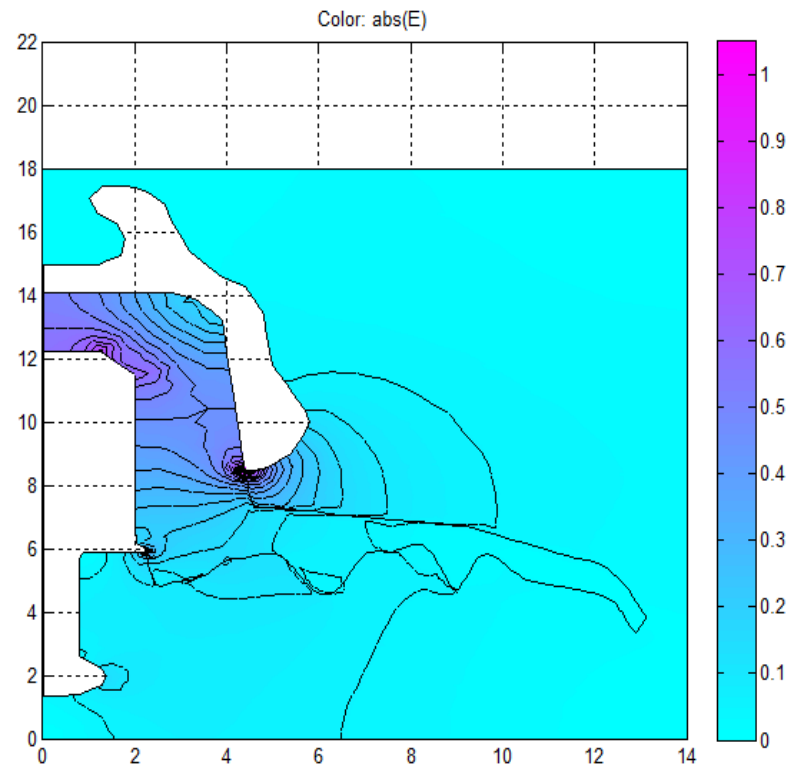

Fig.4 optimal shape of insulator obtained from HGAPSO $\left(a_{3}=0.166\right)$ 


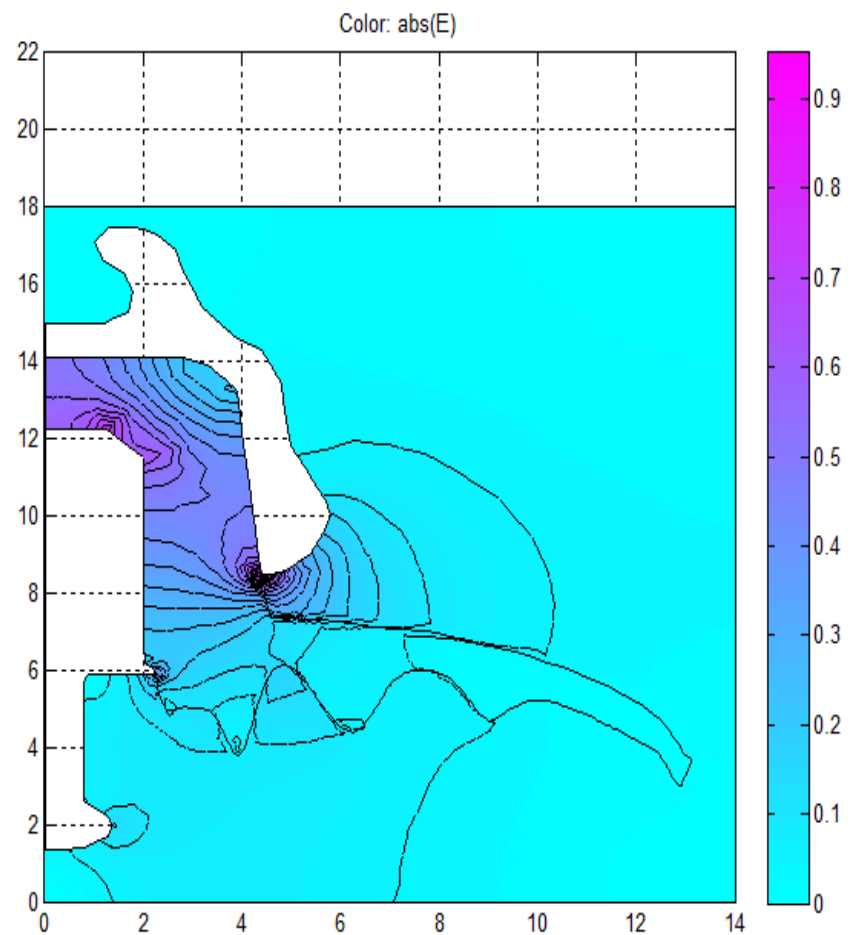

Fig.5 optimal shape of insulator obtained from GA $\left(a_{3}=0.166\right)$

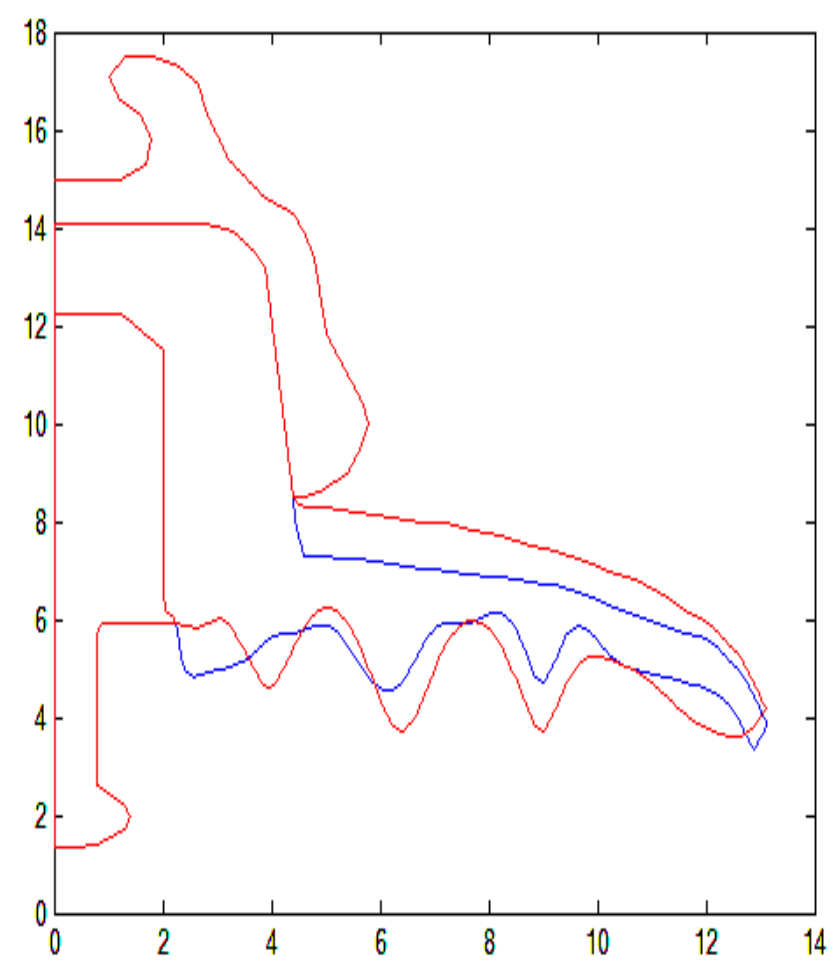

Fig.6 comparison of the optimal shape obtained from HGAPSO with the original shape $\left(a_{3}=0.166\right)$

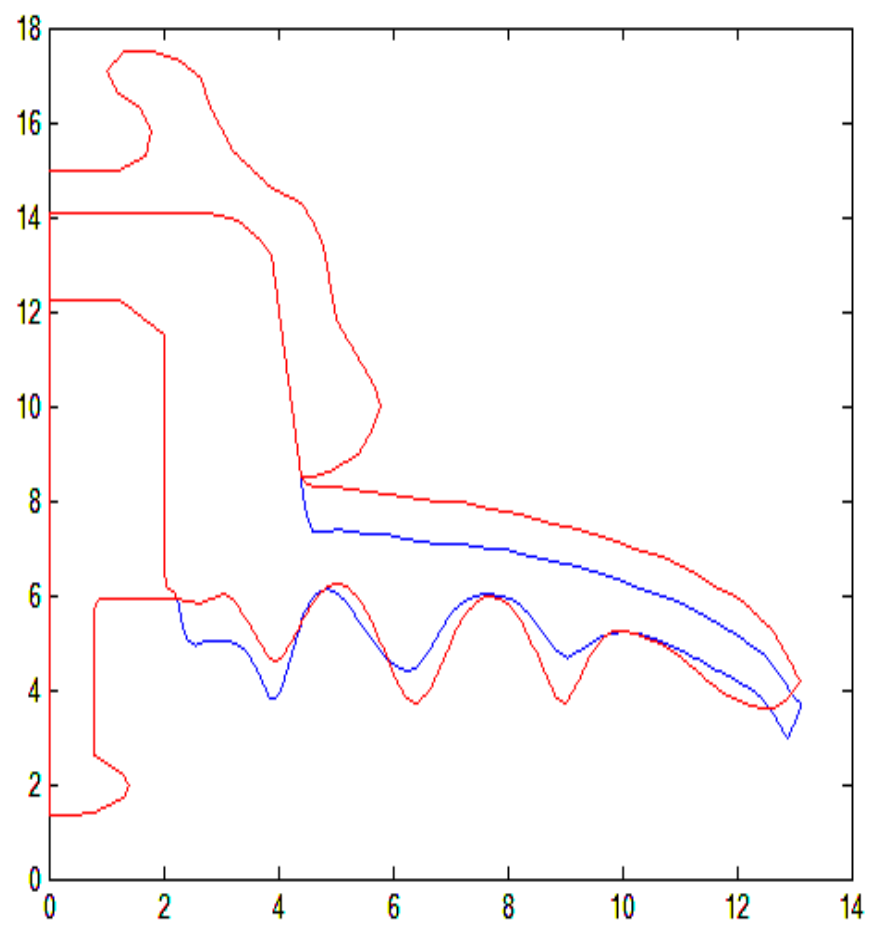

Fig.7 comparison of the optimal shape obtained from GA with the original shape $\left(a_{3}=0.166\right)$

TABLE I

COMPARISON OF THE RESULTS OBTAINED FROM GA and HGAPSO WITH THE VALUES OF ORGINAL INSULATOR $\left(\mathrm{a}_{3}=0.166\right)$

\begin{tabular}{crcc}
\hline & GA & HGAPSO & $\begin{array}{c}\text { Original } \\
\text { Insulator }\end{array}$ \\
$E_{\text {ave }}$ & 0.0618 & 0.0628 & 0.0949 \\
$\sigma_{E}$ & 0.0494 & 0.0489 & 0.1226 \\
$A_{s}$ & 21.0240 & 19.0207 & 28.3146 \\
\hline
\end{tabular}

The results obtained from HGAPSO and GA for $y_{\text {Min }}=2.45$ $\mathrm{cm}$ and $a_{3}=0$ are presented in Fig 8 and 9, respectively. The optimal design of of insulator obtained from HGAPSO and GA which are shown in Fig 10-11 are compared with original insulator in Fig 12-13, respectively. The minimum of objective function obtained from HGAPSO is equal to 10.7081 which are smaller than the results obtained from GA that is equal to 10.997 .

The average and variance of tangential electric field and the area of insulator are compared for different cases in Table 2. 


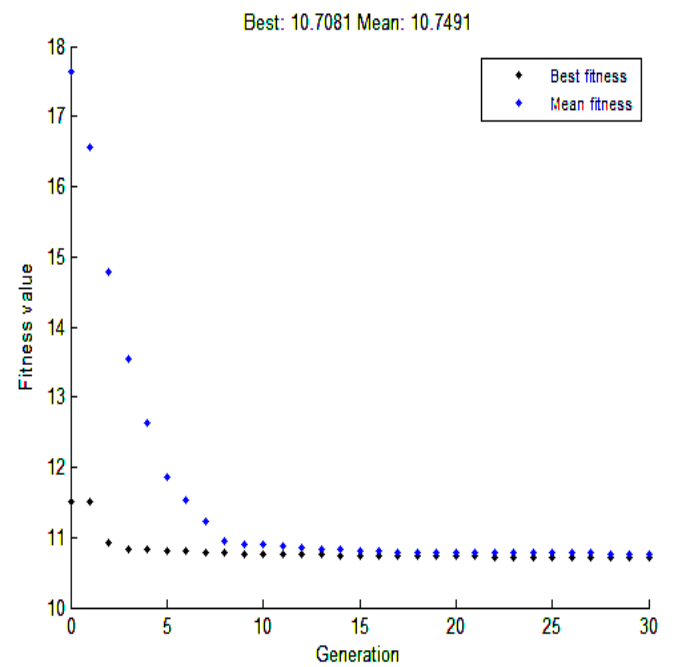

Fig. 8 the minimum values of objective function obtained from HGAPSO $\left(a_{3}=0\right)$

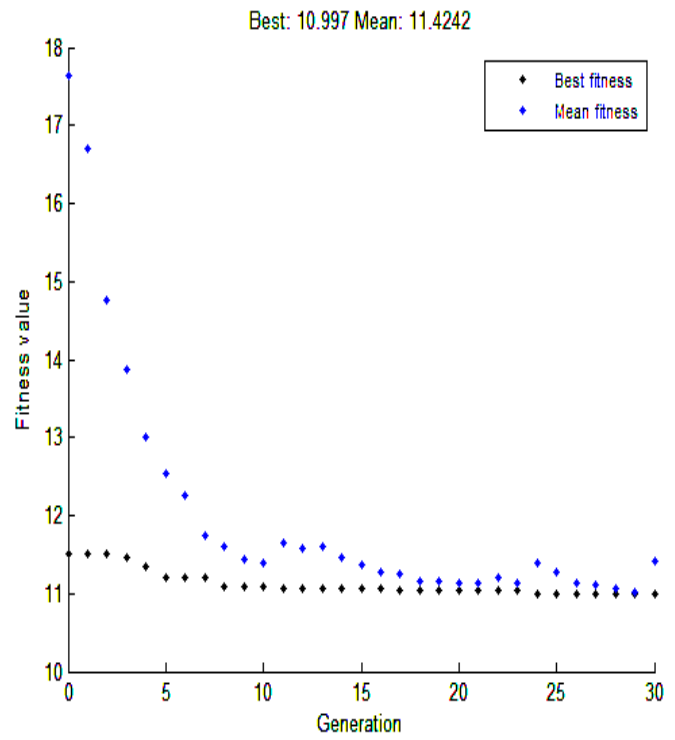

Fig.9 the minimum values of objective function obtained from GA $\left(a_{3}=0\right)$

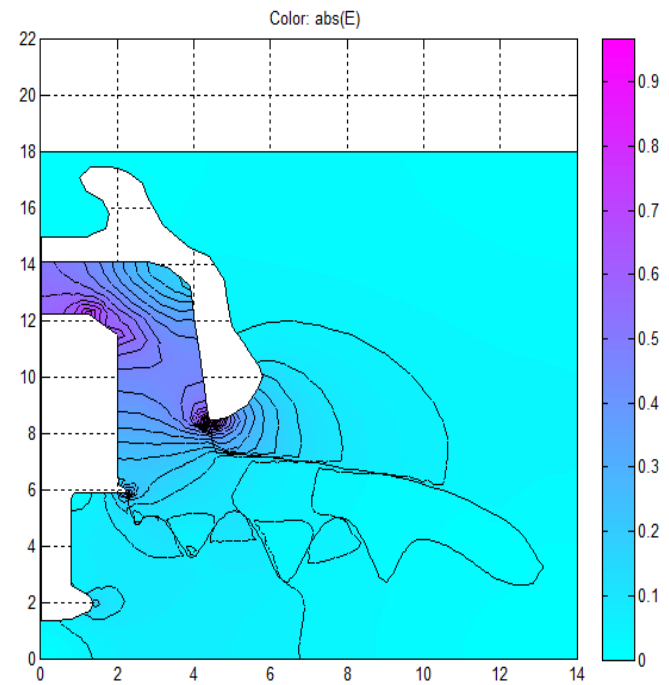

Fig.10 optimal shape of insulator obtained from $\operatorname{HGAPSO}\left(a_{3}=0\right)$

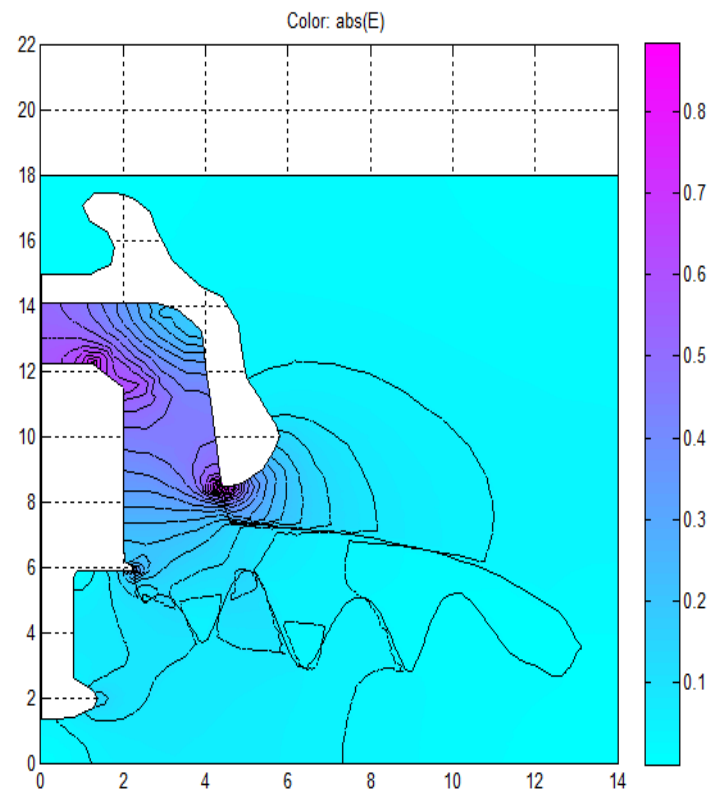

Fig.11 optimal shape of insulator obtained from GA $\left(a_{3}=0\right)$

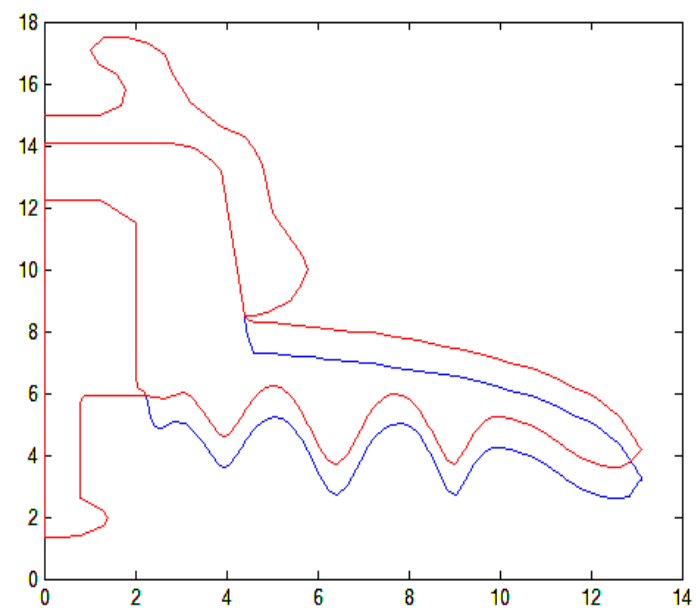

Fig.12 comparison of the optimal shape obtained from HGAPSO with the original shape $\left(a_{3}=0\right)$

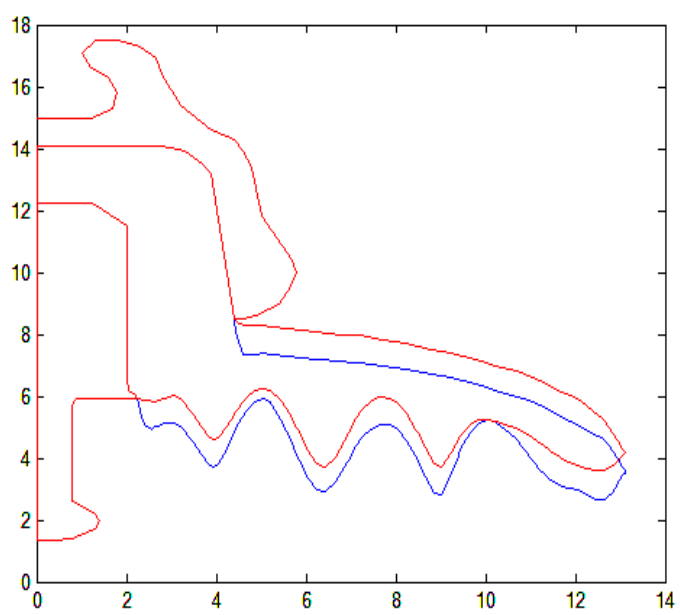

Fig.13comparison of the optimal shape obtained from GA with the original shape $\left(a_{3}=0\right)$ 
TABLE II.

COMPARISON OF THE RESULTS OBTAINED FROM GA and HGAPSO WITH THE VALUES OF ORGINAL INSULATOR $\left(a_{3}=0\right)$

\begin{tabular}{|c|c|c|c|}
\hline & GA & HGAPSO & $\begin{array}{l}\text { Original } \\
\text { Insulator }\end{array}$ \\
\hline$E_{\text {ave }}$ & 0.0599 & 0.0588 & 0.0949 \\
\hline$\sigma_{E}$ & 0.0501 & 0.0483 & 0.1226 \\
\hline$A_{s}$ & 28.8557 & 30.6432 & 28.3146 \\
\hline
\end{tabular}

\section{CONCLUSION}

The minimum of objective function obtained from HGAPSO is smaller than GA. This shows the total coefficients of average and variance of electric field and the area of insulator obtained from HGAPSO is smaller than the result of GA. Therefore, HGAPSO is better than GA for optimizing the shape of insulator. Also, the average and variance of electric field of control points obtained from HGAPSO are smaller than the values of original shape. This shows, we can use HGAPSO for enhancing the distribution of electric field on the insulator.

\section{REFERENCES}

[1] M. Abdel-Salam and E. K. Stanek, "Optimizing field stress of highvoltageinsulators," IEEE Trans. Electr.Insul., vol. 22, no. 1, pp. 47-56,Feb. 1987.

[2] M. Abdel-Salam and E. K. Stanek, "Field optimization of highvoltageinsulators," IEEE Trans. Ind. Appl., vol. IA-22, no. 4, pp. 594601, Jul.1986.

[3] Z. Stih, "High voltage insulating system design by application of electrodeand insulator contour optimization," IEEE Trans. Elect.Insul.,vol. 21, no. 4, pp. 579-584, Aug. 1986.

[4] H. Singer, H. Steinbigler, and P. Weiss, "A charge simulation method for the calculation of high voltage fields," IEEE Trans. Power App.Syst., vol. 126, no. 1, pp. 1660-1668, Feb. 1974.

[5] P. K. Mukherjee and C. K. Roy, "Computation of fields in and around insulators by fictitious point charges," IEEE Trans. Electr. Insul., vol.EI-13, no. 1, pp. 24-31, Feb. 1978.

[6] N. H. Malik, "A review of the charge simulation method and its applications," IEEE Trans. Elect. Insul., vol. 24, no. 1, pp. 3-19, Feb. 1989.

[7] H. El-Kishky and R. S. Gorur, "Electric potential and field computation along ac HV insulators," IEEE Trans. Dielectr.Elect.Insul., vol. 1, no. 6, pp. 982-990, Dec. 1994.

[8] V. Cingoski, K. Kaneda, H. Yamashita, and N. Kowata, "Inverse shape optimization using dynamically adjustable genetic algorithms," IEEETrans. Energy Convers., vol. 14, no. 3, pp. 661-666, Sep. 1999.

[9] R. Hinterding, H. Gieleski, and T. C. Peachey, "The nature of mutation in genetic algorithm," in Proc. 6th Int. Conf. Genetic Algorithms, Jul.1995, pp. 65-72.

[10] W. Chen, H. Yang, and H. Huang, "Contour Optimization of Suspension Insulators Using Dynamically Adjustable Genetic Algorithms," IEEE Trans. Power Delivery, vol. 25, no. 3, pp. 12201228, Jul. 2010.

[11] O. W. Andersen, "Finite element solution of complex potential electric fields," IEEE Trans. Power App. Syst., vol. PAS-96, no. 4, pp.11561161, Jul./Aug. 1977.

[12] MATLAB PDE Toolbox User's Guide, Version 7.6, 2008.

\section{BIOGRAPHIES}

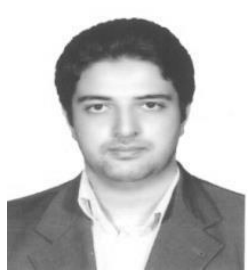

MOHAMMAD REZA SALIMAN was born in Tehran, Iran, in 1986. He received the B.Sc. degree from the Semnan University, Semnan, Iran, MSc degree from Imam Khomeini International University, Qazvin, Iran, and Ph.D. degree from Shahid Beheshti University, Tehran, Iran, all in electrical engineering. His research interests are power system protection and dynamics.

HAMID JAVADI is with the Department of Electrical Engineering, Faculty of Shahid Abbaspour, Shahid Beheshti University, Tehran, Iran. His research interest is power system protection. 